Hans-Jörg Oestern

Klinik für Unfall- und Wiederherstellungschirurgie, Allgemeines Krankenhaus Celle

\title{
Aktueller Stand der Behandlung von Radiusfrakturen
}

rotz der Häufigkeit der distalen Radiusfraktur gibt es keine verbindlichen Therapieempfehlungen. In einem Cochrane-Review wurden randomisierte oder quasirandomisierte klinische Studien für Erwachsene mit distalen Radiusfrakturen analysiert, die die operativen und konservativen Behandlungen verglichen sowie die unterschiedlichen Versorgungsformen oder die Dauer der Immobilisation postoperativ untersuchten. Den Einschlusskriterien des Review mit insgesamt 3193 Frakturen entsprachen 44 Untersuchungen, die 23 Behandlungsformen verglichen. Bei 44 randomisierten Untersuchungen konnte keine Evidenz für die Entscheidungsfindung der unterschiedlichen Behandlungsformen nachgewiesen werden.

Die distalen Radiusfrakturen machen etwa 14\% aller Extremitätenverletzungen aus. Bei älteren Patientengruppen erleiden mehr Frauen als Männer Frakturen des distalen Radius. Das Verhältnis zwischen distalen Radiusfrakturen und Osteoporose wurde von Earnshaw et al. [3] evaluiert: Unter 106 von 149 konsekutiv untersuchten Frauen mit distalen Radiusfrakturen jenseits der Menopause hatte die Hälfte eine Osteoporose der Wirbelsäule, des proximalen Femurs oder des Radius. Patienten unter 65 Jahren hatten eine signifikant geringere Knochendichte im Bereich der Hüfte im Vergleich zu altersbezogenen Werten. Ähnliche Werte fanden Wigderowitz et al. [17] bei 31 Frauen mit distaler Radiusfraktur im Vergleich zu einer Kontroll- gruppe ähnlich alter Frauen. Eine geringere Knochendichte, die mehr als eine Standardabweichung unterhalb der Kontrollgruppe lag, hatten 25 der 31 Frauen.

Diese Tatsache hat die WHO zu der Empfehlung veranlasst, dass Frakturen des distalen Radius bei Frauen in der Postmenopause eine Indikation für die Bestimmung der Knochendichte darstellen und dass die nationale amerikanische Osteoporosestiftung die Knochendichtemessung für alle Frauen mit einer distalen Radiusfraktur jenseits der Menopause empfiehlt.

Freedman et al. [4] analysierten eine nationale Versicherungsdatenbank, um die Diagnostik oder Behandlung der Osteoporose innerhalb von 6 Monaten bei distalen Radiusfrakturen unter Frauen jenseits des 55. Lebensjahrs zu bestimmen. Von 1162 Patienten, die in diese Studie eingeschlossen wurden, hatten sich weniger als $3 \%$ einer Knochendichtemessung unterzogen, und weniger als $23 \%$ wurden mit wenigstens einem Medikament gegen Osteoporose behandelt (Östrogene, Biphosponate oder Kalzitonin).

C) Springer-Verlag 2003

Prof.Dr. Hans-Jörg Oestern

Klinik für Unfall- und

Wiederherstellungschirurgie,

Allgemeines Krankenhaus Celle,

Siemensplatz 4, 29223 Celle

E-Mail: hans-joerg.oestern@akh-celle.de, Phone: 05141-721100, Fax: 05141-721109 


\section{H.-J.Oestern}

\section{Present status of treatment for radial fractures}

\begin{abstract}
The treatment of distal fracturs of the radius has undergone a considerable change in recent years, from conservative treatment through fixation with Kirschner wires to plate fixation. Plae fixation involving stabilisation with fixed-angle implants has proved to lead to few complications. So far, fixed-angle implants have been used for 39 fractures in patients of the author's own. Bone healing was obtained in all these cases, and no complications were observed.
\end{abstract}

\section{Keywords}

Distal fracture of the radius - External fixator . Plate osteosynthesis · Osteosynthesis
Vogt et al. [15] untersuchten in einer prospektiven Studie in einem Zeitraum von 9,8 Jahren über 9000 Frauen im Alter von 65 Jahren und älter. Die Ergebnisse zeigten eine Inzidenz von 7,3 auf 1000 Personen/Jahr. Die Verteilung intraartikulärer zu extraartikulären Frakturen betrug $27 \% \mathrm{zu} 73 \%$. Einflussfaktoren waren eine verminderte Knochendichte (pro $0,1 \mathrm{~g} / \mathrm{cm}^{2}$ ) im Bereich des distalen Radius mit einem relativen Risiko von 1,8. Bei häufigen Stürzen betrug das relative Risiko 1,6 und bei einer stattgehabten Fraktur jenseits des 50. Lebensjahrs 1,3. Dagegen reduzierte die Östrogeneinnahme das relative Risiko $(0,6)$. Bei Frauen mit Diabetes mellitus entstanden doppelt so häufig intraartikuläre Frakturen.

\section{Radiologische Untersuchung}

Die richtige Einschätzung der intraartikulären Frakturen ist entscheidend. Knirk u. Jupiter [10] definierten eine Gelenkstufe von $2 \mathrm{~mm}$ oder mehr als Präarthrose.

Trumble et al. [14] evaluierten die Faktoren, die den Outcome bei verschobenen intraartikulären Frakturen terminieren, und fanden eine entscheidende Verschlechterung der funktionellen Ergebnisse bei einer postoperativen Diastase von mehr als $1 \mathrm{~mm}$ in den postoperativen Röntgenbildern. Die Möglichkeit, Röntgenbilder bis zu diesem Genauigkeitsmaß zu bestimmen, wurde ebenfalls untersucht. Kreder et al. [11] analysierten 16 Untersucher mit unterschiedlichem Ausbildungsstand, um die Intra- und Interobservergenauigkeit verheilter Frakturen auf Standardröntgenbildern zu untersuchen. Während die extraartikulären Messungen einschließlich Ulnavorschub und Palmarinklinationswinkel eine höhere Übereinstimmungsrate aufwiesen, bestanden bei intraartikulären Untersuchungen sogar bei sehr erfahrenen Klinikern erhebliche Unterschiede. Bei der Bestimmung der Stufen und Diastasen unterschieden sich 2 Untersucher in $10 \%$ der Fälle um mehr als $3 \mathrm{~mm}$, und auch wiederholte Messungen durch denselben Untersucher waren in $10 \%$ der Fälle um mehr als $2 \mathrm{~mm}$ verschieden. Die Unterscheidung von 1-mm-Stufen wurde häufiger inkorrekt als korrekt beschrieben. Eine ähnlich schlechte Interobserverübereinstimmung fanden Cole et al. [2] mit einer 30\%igen Über- oder Unterschätzung der Dislokation im Vergleich zum CT. Als geeignete Methode zur Bestimmung einer Gelenkverschiebung wurde deshalb das CT empfohlen.

Bozentka et al. [1] konnten keine Unterschiede in der Inter- und Intraobserverreliabilität im Vergleich zwischen normalen und digitalisierten Röntgenaufnahmen beobachten.

\section{Behandlung}

Die Behandlung der distalen Radiusfrakturen ist kontrovers. Es gibt keine einzige definitive Behandlungsmethode,
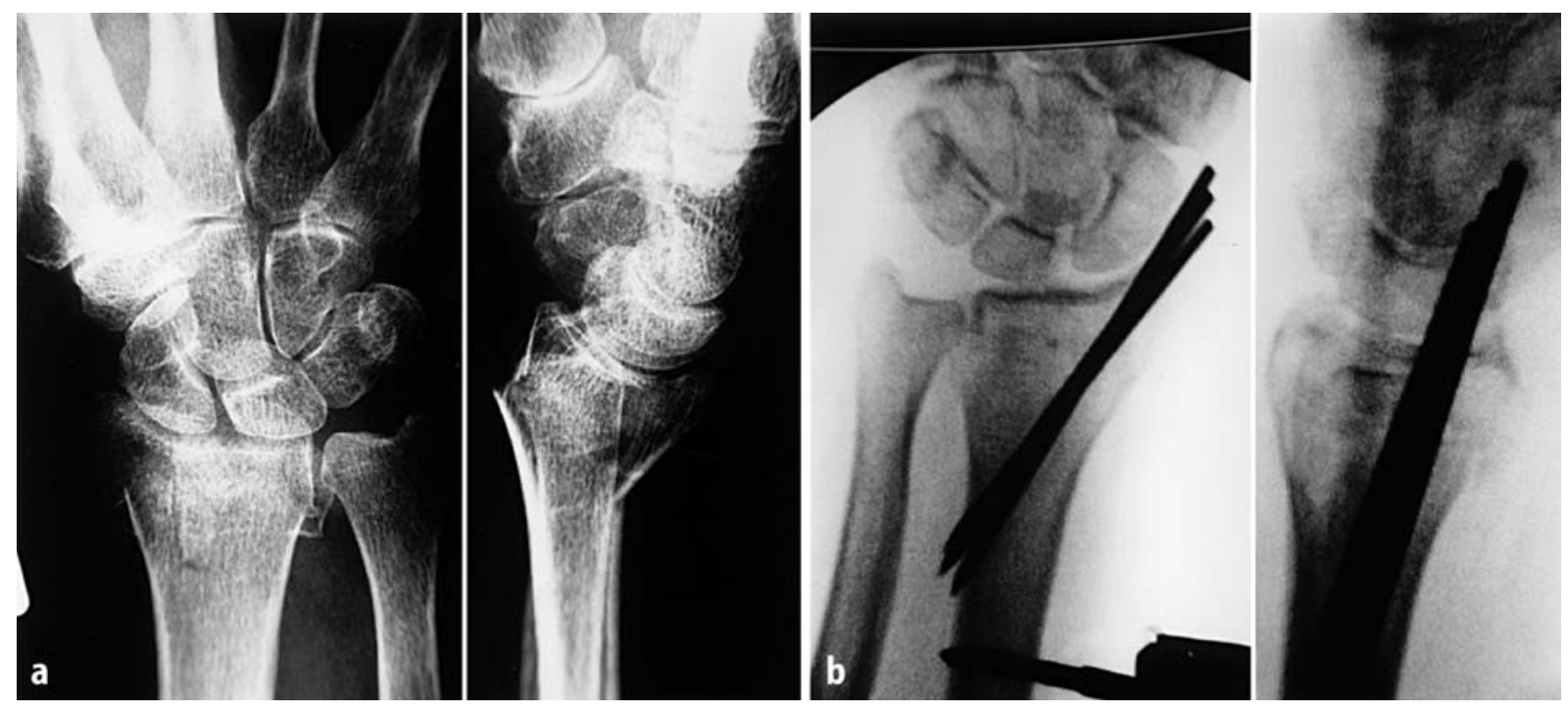

Abb. 1a,b $\Delta$ Kombiniertes Vorgehen mit Fixateur und Kirschner-Drähten, a Unfallbilder, b Versorgungsbilder 
die als Standardmethode gelten kann. Die meisten Studien sind retrospektive Analysen und verwenden verschiedene Klassifikationen und nicht vergleichbare Outcomeuntersuchungen speziell bei Trümmerfrakturen mit Gelenkinkongruenz.

Kreder et al. [11] zeigten die Resultate einer prospektiv randomisierten Multicenterstudie mit 147 Patienten unter 75 Jahren mit dislozierten intraartikulären distalen Radiusfrakturen. Die Patienten wurden randomisiert in 2 Gruppen unterteilt.

\section{- Gruppe I}

Patienten dieser Gruppe wurden mit indirekter Reposition, perkutaner Kirschner-Draht-Fixation mit oder ohne begleitenden Fixateur behandelt.

- Gruppe II

Bei dieser Gruppe erfolgte eine offene Reposition mit Spongiosaplastik.

Die Untersuchungen in diesen beiden Gruppen zeigten, dass nach 6 Monaten Patienten mit indirekter Reposition und perkutaner Stabilisierung eine signifikant bessere Funktion aufwiesen als Patienten mit offener Reposition und interner Fixation.

Kapoor et al. [9] konnten bei einem Follow up von durchschnittlich 4 Jahren über ähnliche Resultate bei über $90 \mathrm{~Pa}$ tienten mit geschlossenen intraartikulären Frakturen berichten, die randomisiert mit geschlossener Reposition und Fixa- teur externe oder offener Reposition und interner Fixation behandelt wurden.

\section{Fixateur externe}

Frykman et al. [5] analysierten 13 Fixateur-externe-Modelle hinsichtlich Gewicht, Kosten, Steifigkeit in verschiedenen Ebenen und ihrer verschiedenen Applikationsmöglichkeiten. Das Ergebnis ihrer Untersuchungen bestand darin, dass die Auswahl eines spezifischen Fabrikats meist von der einfachen Applikationstechnik und der Gewöhnung des Chirurgen mit dem Fixateur abhängt. Die Ansicht, dynamische Fixateure seien überlegen, wurde durch Studien widerlegt, die eine Nachsinterung mit dorsaler Fehlstellung und fast 20\% mäBige oder schlechte radiographische $\mathrm{Re}$ sultate nachwiesen. Wolfe et al. $[18,19]$ und Weiland [16] zeigten, dass eine zusätzliche Kirschner-Draht-Fixation die Beweglichkeit im Bereich der Fraktur reduziert und die Stabilität extraartikulärer Frakturen verbessert.

\section{Kirschner-Draht-Fixation}

\section{Perkutane Kirschner-Draht-Fixation}

Verschiedene Konfigurationen der perkutanen Kirschner-Draht-Fixation sind für die Stabilisierung distaler Radiusfrakturen empfohlen worden [13]. Die perkutane Kirschner-Draht-Fixation wurde verwendet
- für verschobene extraartikuläre Frakturen mit oder ohne dorsale Trümmerzone oder nach Repositionsverlust bei konservativer Behandlung bzw.

- bei intraartikulären Trümmerfrakturen, wenn eine adäquate geschlossene Reposition nicht ausreichend ist.

\section{Intrafokale Kirschner-Draht-Fixation}

Die intrafokale Kirschner-Draht-Fixation, wie sie von Kapandji [8] propagiert wurde, platziert 1,6-mm-KirschnerDrähte direkt in die Fraktur. Um die ulnare Inklination und die radiale Translation zu korrigieren, sind 1-3 Drähte nötig. Die Resultate bei jüngeren Patienten sind aufgrund der besseren Knochenqualität günstiger. Trumble et al. [14] analysierten ihre Erfahrung mit intrafokaler Fixation mit und ohne Fixateur externe bei 61 Patienten, 23 waren älter als 55 Jahre. Die Resultate zeigten, dass die intrafokale Kirschner-Draht-Fixation bei älteren Patienten funktionell und radiologisch schlechter ist als die intrafokale Kirschner-Draht-Fixation mit Fixateur externe (Abb. 1). Bei jüngeren Patienten wurde durch einen ergänzenden Fixateur externe keine zusätzliche Verbesserung erzielt.

\section{Plattenosteosynthese}

In der Zugangsfrage weist der dorsale Zugang eine erhöhte Irritation der Strecksehnen auf. Eine deutliche Ver-

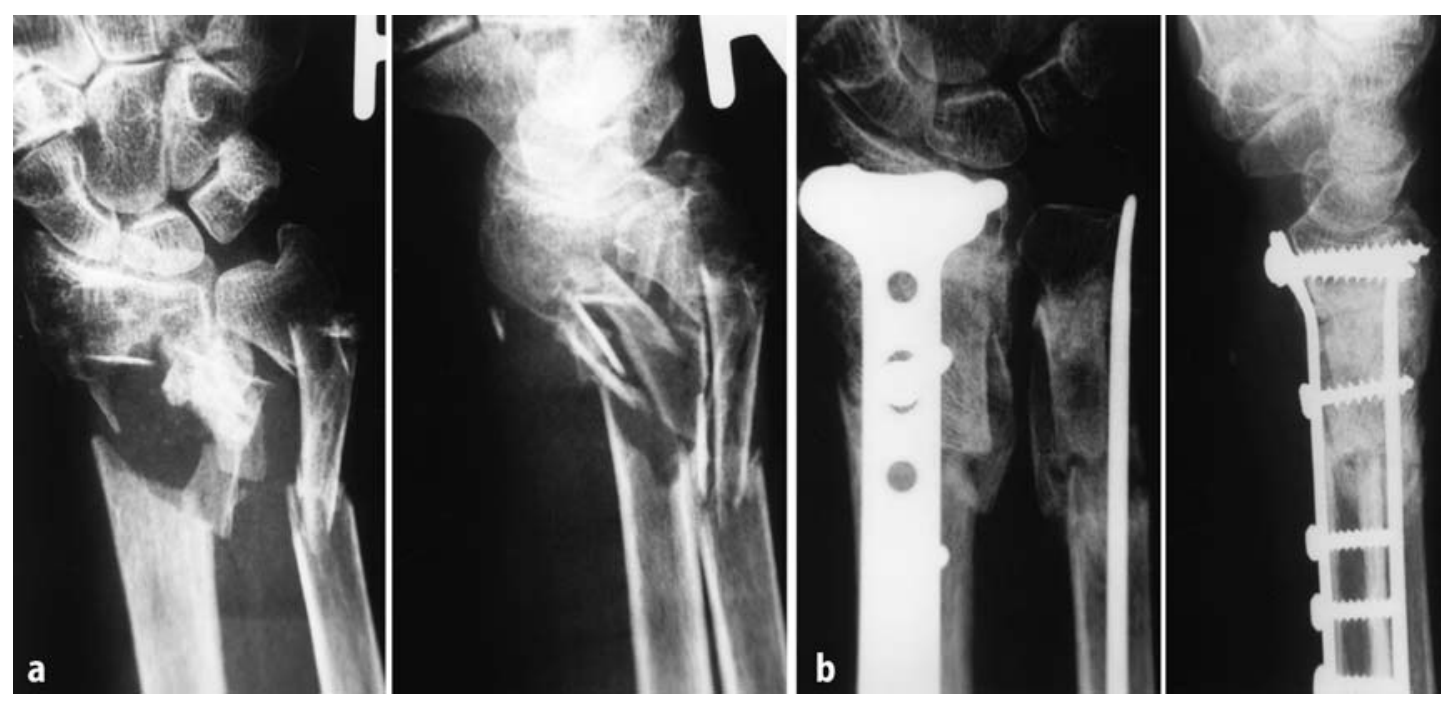

Abb. 2a,b $\Delta$ Defektfraktur und Auffüllung mit kortikospongiösem Span, intramedulläre Schienung der Ulna und volare Plattenosteosynthese, a Unfallbilder, b Versorgungsbilder 

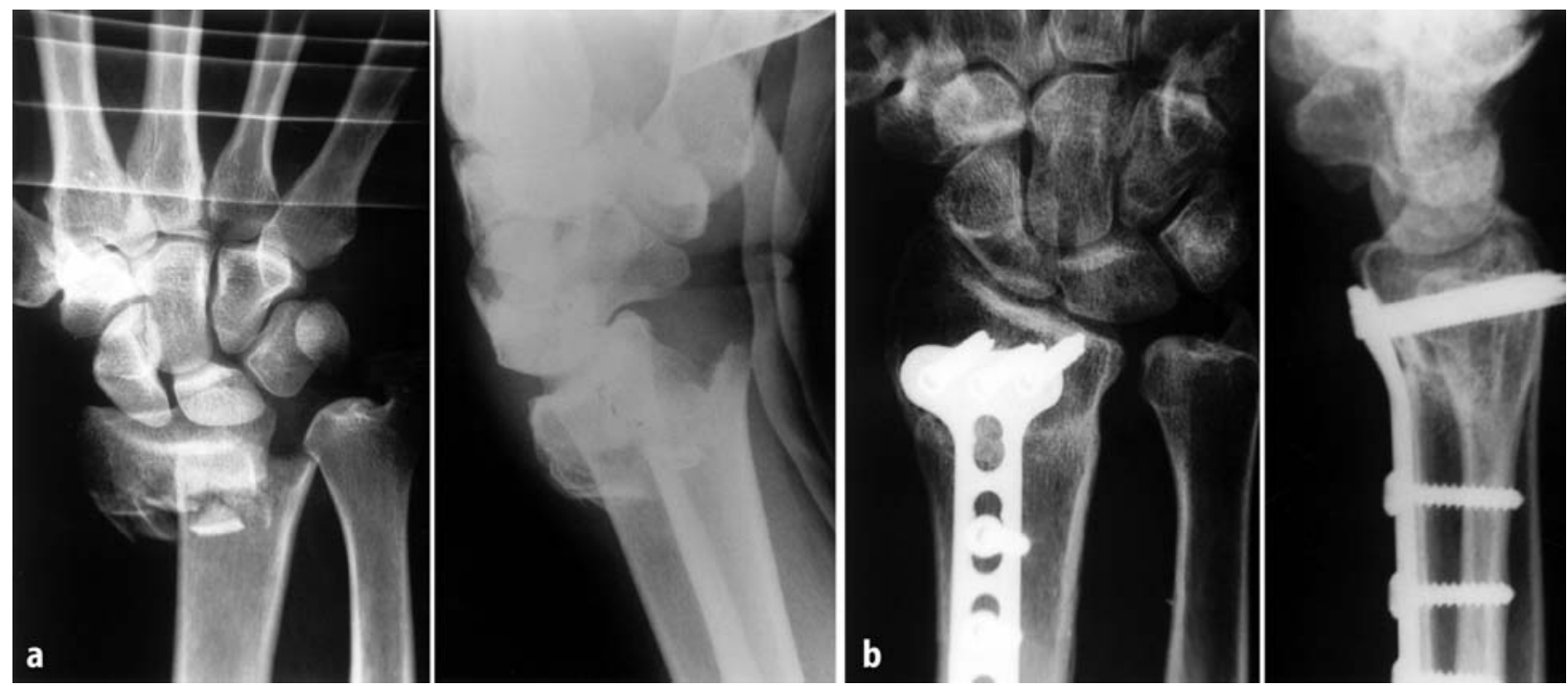

Abb. 3a,b $\Delta$ Versorgung mit winkelstabiler Platte, a Unfallbilder, $b$ Versorgungsbilder

besserung stellt der volare Zugang mit Stabilisierung durch winkelstabile Implantate dar. Über hervorragende Ergebnisse mit der palmaren Plattenosteosynthese berichteten Kamano et al. [7] mit 32 sehr guten und guten und nur 1 schlechten Ergebnis in einer kleineren Serie.

Ähnliche Ergebnisse veröffentlichten Orbay u. Fernandez [12], die in 31 Fällen sehr gute und gute Ergebnisse mit einer volaren Plattenosteosynthese unter Erhalt der dorsalen Weichteilstrukturen mit einer schnelleren Frakturheilung und einer deutlichen niedrigeren Zahl an Spongiosatransplantationen erreichten.

Im eigenen Krankengut wurden bisher 39 Frakturen mit winkelstabilen Implantaten versorgt. Alle Frakturen konnten zur Ausheilung gebracht werden. Aufgrund der geringeren Weichteilirritation bevorzugen wir ebenfalls den volaren Zugang (Abb. 2,3).

\section{Resümee}

Zusammenfassend hat die Behandlung der distalen Radiusfrakturen in den letzten Jahren einen erheblichen Wandel von der konservativen Therapie über die Fixation mittels Kirschner-Drähten bis jetzt zur Plattenosteosynthese durchlaufen. Die Ergebnisse haben sich mit der richtigen Klassifikation und dem jeweils adaptierten Osteosyntheseverfahren entsprechend verbessert.

\section{Literatur}

1. Bozentka DJ, Beredjiklian PK, Westwaski D, Steinberg DR (2002) Digital radiographs in the assessment of distal radius fracture parameters. Clin Orthop 397: 409-413

2. Cole RJ, Bindra RR, Evanoff BA et al. (1997) Radiographic evaluation of osseous displacement following intraarticular fractures of the distal radius: reliability of plain radiography versus computed tomography.J Hand Surg [Am] 22: 792-800

3. Earnshaw SA, Cawte SA, Worley A et al. (1998) Colles' fracture of the wrist as an indicator of underlying osteoporosis in postmenopausal women: a prospective study of bone mineral density and bone turnover rate. Osteoporos Int 8: 53-60

4. Freedman KB, Kaplan FS, Bilker WB et al. (2000) Treatment of osteoporosis: are physicians missing an opportunity? J Bone Joint Surg Am 82: 1063-1070

5. Frykman GK, Peckham RH, Willard K et al. (1993) External fixators for treatment of unstable wrist fractures: a biomechanical, design feature, and cost comparison. Hand Clin 9: 555-565

6. Geissler WB (1995) Arthoroscopically assisted reduction of intra-articular fractures of the distal radius. Hand Clin 11: 19-29

7. Kamano M, Honda Y, Kazuki K, Yasuda M (2002) Palmar plating for dorsally displaced fractures of the distal radius. Clin Orthop 397: 403-408

8. Kapandji A (1987) Internal fixation by double intrafocal pinning: functional treatment of non-articular fractures of the lower end of the radius. Ann Chir Main 6: 57-63

9. Kapoor H, Agarwal A, Dhanon BK (2000) Displaced intraarticular fractures of distal radius: a comparative evaluation of results following closed reduction, external fixation and open reduction with internal fixation. Injury 31 : 75-79
10. Knirk JL, Jupiter JB (1986) Intra-articular fractures of the distal end of the radius in young adults.J Bone Joint Surg Am 68: 647-659

11. Kreder H, Stephen D, Axelod T et al. (1999) A randomised controlled trial of indirect reduction and percutaneous fixation versus ORIF for displaced intraarticular distal radius fractures. American Academy of Orthopaedics Surgeons, $66^{\text {th }}$ Annual Meeting, Anaheim, CA

12. Orbay JL, Fernandez DL (2002) Volar fixation for dorsally displaced fractures of the distal radius: a preliminary report.J Hand Surg [Am] 27: 205-215

13. Rayhack JM (1993) The history and evolution of percutaneous pinning of displaced distal radius fractures. Orthop Clin North Am 24: 287-300

14. Trumble TE, Wagner W, Hanel DP et al. (1998) Intrafocal (Kapandji) pinning of distal radius fractures with and without external fixation. J Hand Surg [Am] 23: 381-394

15. Vogt MT, Cauley JA, Tomanino MM, Stone $K$, Williams JR, Herndon JH (2002) Distal radius fractures in older women: a 10-year-follow-up study of descriptive characteristics and risk. J Am Geriatr Soc 50: 97-103

16. Weiland AJ (1999) External fixation, not ORIF, as the treatment of choice for fractures of the distal radius. J Orthop Trauma 13: 570-572

17. Wigderowitz CA, Rowley DI, Mole PA et al. (2000) Bone mineral density of the radius in patients with Colles fracture.J Bone Surg Br 82: 87-89

18. Wolfe SW, Swigart CR, Grauer J et al. (1998) Augmented external fixation of distal radius fractures: a biomechanical analysis.J Hand Surg [Am] 23: 127-134

19. Wolfe SW, Austin G, Lorenze M et al. (1999) Biomechanical comparison of different wrist external fixators with and without K-wire augmentation.J Hand Surg [Am] 24: 516-524 